\title{
Editorial
}

\section{Editorial: Guided-Wave Optics}

\author{
Boris A. Malomed ${ }^{1,2}$ (iD \\ 1 Department of Physical Electronics, School of Electrical Engineering, Faculty of Engineering, \\ Tel Aviv University, Tel Aviv 69978, Israel; malomed@post.tau.ac.il \\ 2 ITMO University, St. Petersburg 197101, Russia
}

Received: 11 September 2017; Accepted: 15 September 2017; Published: 20 September 2017

\section{An Overview of the Topic and Its Ramifications}

\subsection{Introduction}

Guided waves represent a vast class of phenomena in which the propagation of collective excitations in various media is steered in required directions by fixed (or, sometimes, reconfigurable) conduits. Arguably, the most well-known and practically important waveguides are single-mode and multi-mode optical fibers [1,2], including their more sophisticated version in the form of photonic crystal fibers [3] and hollow metallic structures transmitting microwave radiation [4]. Light pipes, in the form of hollow tubes with reflecting inner surfaces, are used in illumination techniques. On the other hand, medical stethoscopes offer a commonly known example of a practically important acoustic waveguide. New directions of studies in photonics are focused on waveguides for plasmonic waves on metallic surfaces [5-7] (which provide the possibility of using wavelengths much smaller than those corresponding to the traditional optical range, and thus offer opportunities to build much more compact photonic devices) and on the other hand, on the guided transmission of terahertz waves, which also have a great potential for applications [8].

Outside of the realm of photonics (optics and plasmonics) and acoustics, wave propagation plays a profoundly important role in many other areas; accordingly, waveguiding settings have drawn a great deal of interest in those areas as well. In particular, as concerns hydrodynamics, natural waveguides - which may be very long-exist for internal waves propagating in stratified liquids (e.g., in the ocean) [9]. Various settings in the form of waveguides for matter waves are well known in studies of Bose-Einstein condensates in ultracold bosonic gases $[10,11]$. In solid-state physics, guided propagation regimes for magnon waves in ferromagnetic media are a subject of theoretical and experimental studies [12]. In superconductivity, long Josephson junctions are waveguides for plasma waves [8,13]. The significance of waveguiding in plasma physics is also well-known; e.g., Ref [14-16].

Below, a very brief overview of basic theoretical models and experimental realizations of various physical implementations of the waveguiding phenomenology is given. The text is structured according to the character of the guided wave propagation: linear or nonlinear and conservative or dissipative, as well as according to the materials used in the underlying settings, natural or artificial.

This presentation definitely does not aim to include an exhaustive bibliography on this vast research area. References are given chiefly to review articles and books summarizing the known results, rather than to original papers where the results were first published. However, in some cases original papers are also cited if it is necessary in the context of the presentation.

\subsection{Linear Waveguides}

The basic waveguiding structure is a single-mode conduit, designed with a sufficiently small transverse size and boundary conditions at the boundary between the guiding core and surrounding cladding, which admits the propagation of a single transverse mode, while all 
higher-order modes get imaginary propagation constants (i.e., they cannot actually propagate). A commonly known - and arguably the most important-example is provided by single-mode optical fibers (although, strictly speaking, all such fibers are bimodal if the polarization of light is taken into consideration) $[17,18]$. Single-mode waveguides are crucially important components of telecommunication systems, while other applications (e.g., the delivery of powerful laser beams for material processing and the creation of complex spatiotemporal patterns) are best served by multimode conduits $[19,20]$.

Parallel to waveguiding fibers, planar waveguides are a subject of many studies in optics. In the corresponding models, as well as in their fiber-optics counterparts, the evolution variable is the propagation distance, $z$; see Equation (2) below (this is a common feature of all guided-wave-propagation settings, not only in optics, but in other physical realizations of waveguides as well). Meanwhile, the transverse coordinate, $x$, in the spatial domain plays the same role as the reduced-time variable,

$$
\tau \equiv t-V_{\mathrm{gr}}^{-1} z,
$$

where $t$ is the time proper, and $V_{\mathrm{gr}}$ is the group velocity of the carrier wave in the temporal domain in fiber optics. The waveguiding structure in the planar waveguide is represented (roughly speaking) by a stripe with a locally increased effective refractive index.

Effective equations which model the temporal-domain propagation of optical waves in fibers and the spatial-domain propagation in planar waveguides are similar to each other, taking the form of the linear Schrödinger equation for local amplitude $u$ of the electromagnetic wave, which is written here in terms of the spatial-domain propagation, and in the scaled form:

$$
i \frac{\partial u}{\partial z}+\frac{1}{2} \frac{\partial^{2} u}{\partial x^{2}}-U(x) u=0 .
$$

In particular, the aforementioned stripe waveguiding channel is represented by trapping potential $U(x)$ in Equation (2), while the second derivative in Equation (2) represents the paraxial (weak) transverse diffraction in the planar waveguide. A ubiquitous form of the potential is

$$
U(x)=-\epsilon \operatorname{sech}^{2}(x / l),
$$

where $\epsilon>0$ determines the effective depth of the potential well, and $l$ determines its width. In the temporal domain, the transverse coordinate, $x$, is replaced by the above-mentioned temporal variable (1). and the diffraction term in Equation (2) is replaced by $-(\beta / 2) \partial^{2} u / \partial \tau^{2}$, where $\beta$ is the coefficient of the group-velocity dispersion $(\beta>0$ and $\beta<0$ correspond to the normal and anomalous dispersion, respectively).

Further, the similarity between the wave-propagation Equation (2) in optics and the Schrödinger equation in quantum mechanics suggests a similarity between the guided transmission of waves in the guiding channel and propagation of real quantum particles in holding channel potentials [21]. The consideration of the transport of quantum particles in such channels gives rise to many intriguing peculiarities, such as the consideration of curved guiding channels. In this context, it is relevant to mention a well-known result which demonstrates a strong effect of the confinement imposed by a pipe-shaped potential on the character of the effectively one-dimensional mutual scattering of two quantum particles, which amounts to full reflection of the colliding particles [22]. This theoretical prediction had suggested the experimental realization of the concept of the Tonks-Girardeau gas; i.e., a gas composed of hard-core bosons, which bounce back from each other when they collide [23,24].

A natural generalization of single-channel waveguides is provided by a coupler, which may be considered as a set of two parallel waveguiding cores, coupled in the transverse direction by tunneling of guided wave fields steered by each tunnel in the longitudinal direction. The respective 
system of coupled equations for amplitudes $u$ and $v$ of electromagnetic waves in the two cores is [25] (cf. Equation (2)):

$$
\begin{aligned}
& i \frac{\partial u}{\partial z}+\frac{1}{2} \frac{\partial^{2} u}{\partial x^{2}}+\kappa v-U(x) u=0 \\
& i \frac{\partial v}{\partial z}+\frac{1}{2} \frac{\partial^{2} v}{\partial x^{2}}+\kappa u-U(x) v=0
\end{aligned}
$$

where $\kappa$ is the coefficient of the linear inter-core coupling.

The next step is to consider arrayed systems, composed of many parallel guiding cores, which are also coupled in the transverse direction(s) by the tunneling of longitudinally guided wave fields (planar and bulk arrays have, respectively, one or two transverse coordinates). The simplest model of such a guiding medium is provided by the two- or three-dimensional scaled Schrödinger equation with a periodic transverse potential, which represents the (idealized) structure of the multi-core bundle:

$$
i \frac{\partial u}{\partial z}+\frac{1}{2}\left(\frac{\partial^{2} u}{\partial x^{2}}+\frac{\partial^{2} u}{\partial y^{2}}\right)-\epsilon\left[\cos \left(\frac{2 \pi x}{l}\right)+\cos \left(\frac{2 \pi y}{l}\right)\right] u=0 .
$$

Here $l$ is the array's period (defined in scaled units, in which Equation (5) is written), and $2 \epsilon$ is the scaled depth of the effective trapping potential. In particular, in optics bulk arrays have been created as permanent structures by burning (also by means of an optical technology) a large number of parallel guiding cores in a bulk piece of silica [26]. As concerns planar guiding arrays, an interesting ramification of the topic is the propagation of optical waves in such arrays made with a curved shape [27]. On the other hand, a technology for the creation of reconfigurable virtual conduit patterns in the form of photonic lattices was elaborated for photorefractive materials [28]. The latter technology makes use of the fundamental property of the photorefractive materials, in which the propagation conditions for light with ordinary and extraordinary polarizations are linear and nonlinear, respectively. To create a photonic lattice, the experimentalist first illuminates the sample by counterpropagating pairs of mutually coherent laser beams in the ordinary polarization, which create a classical interference pattern in the photorefractive crystal, which is an effectively linear medium for these beams. Next, a probe beam is launched, with the extraordinary polarization in the transverse direction. Due to its inherent nonlinearity, the probe beam is affected by the originally created photonic lattice, as if it is a material structure that creates a spatially periodic modulation of the local refractive index in the transverse directions; i.e., essentially, another version of the multi-core guiding structure.

The propagation of light or waves of a different physical nature in arrays with weak coupling between guiding cores may be naturally approximated by the discrete Schrödinger equation. The basic realization of such a medium is represented by planar arrays of parallel optical waveguides coupled by evanescent waves penetrating dielectric barriers separating individual cores, the basic model being a scaled discrete version of Equation (2):

$$
i \frac{d u_{n}}{d z}+\frac{1}{2}\left(u_{n+1}+u_{n-1}-2 u_{n}\right)-U_{n} u_{n}=0,
$$

where the discrete coordinate, $n$, which replaces $x$, is the number of the guiding core in the array. The study of light propagation in various multi-core systems-which may be approximated by lattice models similar to Equation (6) —is a vast area known as discrete optics [29].

\subsection{Nonlinear Waveguides}

In many situations, tightly confined guided waves propagating in conduits with a small effective cross-sectional area acquire high amplitudes, which is a source of a great many fascinating nonlinear 
effects. In particular, waveguides often provide a combination of the nonlinearity, group-velocity dispersion, and low (or sometimes completely negligible) losses which are necessary ingredients for the creation of solitons (robust self-trapped solitary waves). The simplest and ubiquitous model of the nonlinear wave propagation is based on the nonlinear Schrödinger equation (NLSE), which in the simplest case includes a cubic term. In optics, this term represents the Kerr effect; i.e., nonlinear self-focusing (or, sometimes, self-defocusing) of light in the dielectric medium. The accordingly amended linear Schrödinger Equation (2) becomes the NLSE:

$$
i \frac{\partial u}{\partial z}+\frac{1}{2} \frac{\partial^{2} u}{\partial x^{2}}-U(x) u+\sigma|u|^{2} u=0
$$

where $\sigma=+1$ and -1 corresponds, respectively, to the self-focusing and defocusing nonlinearity; i.e., self-attraction and self-repulsion of light in the nonlinear medium. Equations (4) and (5) each acquire the same cubic terms as in Equation (7). In particular, the nonlinear version of Equation (4),

$$
\begin{aligned}
& i \frac{\partial u}{\partial z}+\frac{1}{2} \frac{\partial^{2} u}{\partial x^{2}}+\kappa v-U(x) u+\sigma|u|^{2} u=0 \\
& i \frac{\partial v}{\partial z}+\frac{1}{2} \frac{\partial^{2} v}{\partial x^{2}}+\kappa u-U(x) v+\sigma|v|^{2} v=0
\end{aligned}
$$

is the basic model of nonlinear couplers, their remarkable property being spontaneous symmetry breaking in the case of self-focusing in the parallel-coupled cores, $\sigma=+1[25,30,31]$.

A remarkable property of the one-dimensional NLSE in the absence of the potential $(U=0$ in Equation (7)) is that it is an integrable equation for which a very large number of exact solutions-including multi-soliton states — can be produced by means of a mathematical technique based on the inverse scattering transform [32-34]. These are bright and dark solitons in the cases of self-focusing and defocusing, respectively. In particular, the exact bright-soliton solution to Equation (7) with $\sigma=+1$ and $U=0$ is

$$
u(x, z)=\eta \exp \left(\frac{i}{2}\left(\eta^{2}-c^{2}\right) z+i c x\right) \operatorname{sech}(\eta(x-c z),
$$

where $\eta$ and $c$ are, respectively, the arbitrary amplitude and velocity of the soliton (in fact, in the spatial domain - in terms of which Equation (7) is written - the soliton represents a self-trapped light beam, and accordingly $c$ is not a velocity, but rather a parameter which determines the tilt of the beam in the $(x, z)$ plane).

The discrete Schrödinger Equation (6) also has its natural nonlinear counterpart in the form of discrete NLSE:

$$
i \frac{d u_{n}}{d z}+\frac{1}{2}\left(u_{n+1}+u_{n-1}-2 u_{n}\right)-U_{n} u_{n}+\sigma\left|u_{n}\right|^{2} u_{n}=0
$$

i.e., a discrete version of NLSE (7). The discrete NLSE gives rise to discrete solitons and their bound states, which cannot be found in an exact form, but may be efficiently produced by numerical and approximate analytical methods [35]. The propagation of nonlinear waves in discrete waveguiding arrays was the subject of numerous theoretical and experimental works [29,36].

The multidimensional extension of the NLSE also has direct realizations in optics, as well as in the mean-field model of atomic Bose-Einstein condensates (BECs) [37,38], and in many other areas. In particular, the spatial-domain light propagation in bulk media is modelled by the effectively two-dimensional version of Equation (7), with two transverse coordinates $(x, y)$ :

$$
i \frac{\partial u}{\partial z}+\frac{1}{2}\left(\frac{\partial^{2} u}{\partial x^{2}}+\frac{\partial^{2} u}{\partial y^{2}}\right)-U(x, y) u+\sigma|u|^{2} u=0 .
$$


Unlike its one-dimensional counterpart (7), Equation (11) in the free space $(U(x, y)=0)$ is not integrable. It admits formal soliton solutions, looked for as

$$
u(x, y ; z)=\exp (i k z+i S \theta) U_{S}(r)
$$

in terms of the polar coordinates $(r, \theta)$ in the $(x, y)$ plane, where $k>0$ is a real propagation constant, $S=0, \pm 1, \pm 2, \ldots$, is an integer vorticity that may be embedded in the two-dimensional soliton (shaping it as a vortex ring), and $U_{S}(r)$ is a real radial amplitude function satisfying boundary conditions $U_{S}(r) \sim \exp (-\sqrt{2 k} r)$ at $r \rightarrow \infty$, and $U(r) \sim r^{|S|}$ at $r \rightarrow 0$. Solitons (12) with $S=0$ are often called Townes solitons [39]. However, the Townes solitons-as well as their vortex counterparts, with $S \neq 0$ in Equation (12) - are completely unstable, being vulnerable to destruction by the critical collapse (formation of a singularity after a finite propagation distance) in the case of $S=0$, and by a still stronger instability which splits vortex rings with $S \neq 0$ [39].

An important example of nonintegrable one-dimensional system modelling nonlinear light propagation in optics is the system of coupled-mode equations which describe the fiber Bragg gratings (i.e., nonlinear optical fibers with a periodic lattice of local defects permanently written in their cladding, with a period equal to half the wavelength of light coupled into this waveguide). The coupled-mode equations govern the evolution of amplitudes $u$ and $v$ of right- and left-traveling waves, which are mutually converted (reflected) into each other by the Bragg grating [40,41]:

$$
\begin{aligned}
& i u_{t}+i u_{x}+\kappa v+\left(\frac{1}{2}|u|^{2}+|v|^{2}\right) u=0, \\
& i v_{t}-i v_{x}+\kappa u+\left(\frac{1}{2}|v|^{2}+|u|^{2}\right) v=0,
\end{aligned}
$$

where $\kappa$ is the Bragg-grating reflectivity, and the group velocity of the light waves in the fiber is scaled to be 1 . This system admits exact solutions in the form of solitons, but it is not an integrable one. Such solitons-moving in the fiber Bragg grating as in the waveguide-have been created in the experiment [42]. Roughly half of the soliton family is stable, and half unstable.

The use of fiber Bragg gratings operating in the linear regime has grown into a large industry with many applications, such as sensors, dispersion compensators, optical buffers, etc. [43].

Another fundamentally important nonlinear model for the guided wave propagation is the one with the quadratic (alias second-harmonic) nonlinearity, instead of the cubic (Kerr) term in NLSE (7). The model is based on the propagation equations for complex amplitudes $u(x, z)$ and $v(x, z)$ of the fundamental and second harmonics [44,45]:

$$
\begin{array}{r}
i u_{z}+\frac{1}{2} u_{x x}+v u^{*}=0, \\
2 i v_{z}-q v+\frac{1}{2} v_{x x}+\frac{1}{2} u^{2}=0,
\end{array}
$$

where $q$ is a real mismatch parameter. Although it is a nonintegrable system, Equations (14) also give rise to solitons, which are generically found in a numerical form. These solitons form a family which is chiefly stable, with a small instability area [44,45].

In BEC models, Equation (7), with evolution variable $z$ replaced by (scaled) time, $t$, is called the Gross-Pitaevskii equation, in which the cubic term represents-in the mean-field approximation-an average effect of collisions between atoms [37,38]. The natural sign of the collision-induced term corresponds to self-repulsion (self-defocusing) (i.e., $\sigma=-1$ in Equation (7)), but for atomic species such as ${ }^{7} \mathrm{Li},{ }^{39} \mathrm{~K}$, and ${ }^{85} \mathrm{Rb}$, the sign may be switched to self-attraction by means of the Feshbach resonance, which is in turn controlled by a magnetic or laser field acting on the experimental setup [46]. 
Theoretical and experimental work with solitons and other diverse nonlinear effects (such as the modulational instability [47] and rogue waves [48,49], shock waves, separation of immiscible components in binary systems, kinks, and domain walls [50], instantons [51], etc.) is a huge research area in many branches of physics [52], including optics [47], matter waves in atomic BECs [53], and BECs of quasi-particles (in particular, excitons-polaritons) [54], plasmas [55], ferromagnetic media [56], long Josephson junctions in superconductivity [57], acoustics [58], etc. In many cases, waveguiding settings offer media in which many species of solitons can be created and/or stabilized if the solitons do not exist (or exist but are unstable) in the respective uniform media. Characteristic examples are various methods elaborated for the stabilization of three-dimensional spatiotemporal solitons ("light bullets" [59]), which are subject to strong instabilities in both two- and three-dimensional uniform media [60-62]. It was demonstrated experimentally that both fundamental spatiotemporal solitons [63] and ones with embedded vorticity [64] can be made stable (in fact, as semi-discrete solitons) in the above-mentioned systems created as bundles of parallel waveguiding cores in bulk silica samples [26]. In fact, the commonly known stability of temporal optical solitons in nonlinear fibers [47] is also an example of the stabilization of a localized mode which is-strictly speaking - a three-dimensional one, with the self-trapping in the temporal (longitudinal) direction induced by the nonlinearity, while the transverse trapping is secured by the fiber's guiding properties, which are not essentially affected by the nonlinearity. Furthermore, the stability of matter-wave solitons in cigar-shaped trapping potentials [53] is provided by a similar mechanism, in spite of a completely different physical nature of the latter setting: the longitudinal self-trapping is induced by the self-attraction of the condensate, due to attractive interactions between atoms, while the confining potential prevents spreading of the condensate's wave function in the transverse directions. Moderate deviation from the effective one-dimensionality essentially affects the shape of the matter-wave solitons, but still relies upon the trapping potential to prevent the collapse of the three-dimensional self-attractive condensate [65].

\subsection{Waveguides Built of Artificial Materials}

The experimental and theoretical results outlined above were obtained in naturally existing media (and, accordingly, theoretical models of such media), or in settings produced by straightforward modifications of natural media, such as the aforementioned multi-core bundled guiding structures burnt in bulk silica $[26,63,64]$.

Still natural-but more unusual-optical materials are photonic crystals (PhCs) [66] and quasicrystals [67,68], as well as PhC-based heterostructures and interfaces [69], and PhC fibers [70-72]; i.e., holey fibers in which inner voids form a $\mathrm{PhC}$ structure in the transverse plane. The difference between the traditional monolithic conduits (which guide light by means of the appropriate transverse profile of the refractive index) and $\mathrm{PhCs}$ is that $\mathrm{PhCs}$ implement the bandgap-guidance principle, steering the transmission of different optical modes according to the spectral bandgap structure, as induced by the underlying crystalline lattice.

Related to PhC fibers are waveguides built as large-radius hollow fibers, with a specially designed multi-layer cladding, which-by means of the Bragg-reflection mechanism (acting in the radial direction)-support the omniguiding regime of the transmission of light in such conduits. As a result, the omniguiding fibers (alias Bragg fibers) may provide a quasi-single regime of the propagation for selected modes, even if the large-area fiber is a multi-mode one. This is possible because all the modes except for the selected one will be suppressed by strong losses [73].

It is relevant to mention that another guidance mechanism is also possible which makes use of lattice structures similar to those underlying PhCs and PhC fibers; however, differently from them, these are nonlinear lattices [74]; i.e., spatially periodic modulations of the local nonlinearity coefficient. Naturally, such nonlinear lattices and their combinations with the usual linear lattices [75] are appropriate for steering nonlinear modes—first of all, solitons [74,75]. 
Furthermore, a new mechanism (thus far elaborated theoretically) for guided transmission of one- and two-dimensional spatial optical solitons, as well as their matter-wave counterparts in BEC, makes use of a purely self-defocusing nonlinearity, growing from center to periphery in the $D$-dimensional space faster than $r^{D}$, where $r$ is the radial coordinate [76]. This scheme was predicted to stabilize a large number of diverse self-trapped (soliton-like) modes, both fundamental ones and complex topologically-organized objects, such as three-dimensional hopfions [77] (i.e., vortex rings with internal twist which carry two independent topological numbers: the vorticity and the twist).

$\mathrm{PhCs}$ and their various modifications may indeed be considered as natural materials because such structures are found in various animals, accounting for their coloration [78]. On the other hand, the recent progress in photonics has produced remarkable results in the form of artificially built media, which exhibit completely novel properties that are not possible in natural media; a very important example is provided by left-handed metamaterials, featuring negative values of the refractive index $[79,80]$. This property may be used for realization of fascinating applications, such as superlensing (which breaks the diffraction limit of imaging [81]), and optical cloaking (lending partial invisibility to small objects [82]). Other well-known examples of purposely designed artificial optical media with extraordinary properties include hyperbolic metamaterials, whose tensors of the dielectric permittivity and/or magnetic permeability feature principal values of opposite signs $[83,84]$, planar metasurfaces $[85,86]$, epsilon-near-zero materials, in which the refractive index nearly vanishes [87], photonic topological insulators [88,89] (which exemplify the area of topological photonics [90]), and others. The use of such media opens numerous possibilities to implement diverse optical effects, including nonlinear ones [91] and guided-wave propagation, in forms that were not known previously (for instance, in the form of the surface waveguiding in photonic topological insulators, which is immune to scattering on defects because the scattering is suppressed by the topology of the guiding system), and are unified under the name of metaoptics [92,93]. Another unifying concept is nanophotonics, the name originating from the fact that many of these materials are assembled of elements with sizes measured on the nanometer scale (which is deeply subwavelength, in terms of optics). One of the fundamentally interesting subjects of nanophotonics is trapping and transmitting light in nanowires. Nanowires are optical filaments (usually made of silicon) whose diameter-measured in nanometers-is much smaller than the wavelength of light, while a typical length may be a few millimeters; one of their important applications is in solar photovoltaic elements [94].

\subsection{Dissipative and Parity-Time Symmetric Waveguides}

The brief discussion of the waveguiding mechanisms given above did not address the presence of losses and the necessity of compensating them by gain. This assumption is valid for relatively short propagation distances, as well as in the case when the compensating gain matches the action of losses so accurately that both factors may be simultaneously neglected in the first approximation. In reality, losses are an inevitably existing gradient in plasmonics and metamaterials, as the respective waveguides are based on metallic elements, which introduce the Ohmic dissipation.

Generally speaking, if the medium is essentially lossy, the above-mentioned index-guiding and bandgap-guiding mechanisms which define the guiding channel(s), respectively, in terms of a transverse profile of the local refractive index, or the transmission-band structure induced by the $\mathrm{PhC}$ or $\mathrm{PhC}$ fiber may be replaced by a gain-guiding scheme in which the signal propagates in a lossy planar or bulk medium along a narrow stripe of gain locally embedded into the medium [95-97].

A recently developed topic which is closely related to the light transmission in dissipative waveguides deals with the parity-time $(\mathcal{P} \mathcal{T})$ symmetry, which implies balance between symmetrically (in space) placed gain and loss elements. A paradigmatic model (it often includes nonlinearity, although 
the $\mathcal{P} \mathcal{T}$ symmetry is by itself a linear property) is represented by NLSE (7), in which the potential is made complex, with real and imaginary parts being, respectively, spatially even and odd ones:

$$
\begin{gathered}
i \frac{\partial u}{\partial z}+\frac{1}{2} \frac{\partial^{2} u}{\partial x^{2}}-\left[U_{r}(x)+i U_{i}(x)\right] u+\sigma|u|^{2} u=0, \\
U_{r}(-x)=U_{r}(x), U_{i}\left(-x_{-}=-U_{i}(x) .\right.
\end{gathered}
$$

Another fundamental realization of the $\mathcal{P} \mathcal{T}$ symmetry in optics and related fields is offered by a coupler, in which one core carries uniformly distributed gain, and the parallel-coupled one is uniformly lossy, the accordingly modified Equation (8) being

$$
\begin{aligned}
& i \frac{\partial u}{\partial z}+\frac{1}{2} \frac{\partial^{2} u}{\partial x^{2}}+\kappa v-U(x) u+\sigma|u|^{2} u=i \gamma u, \\
& i \frac{\partial v}{\partial z}+\frac{1}{2} \frac{\partial^{2} v}{\partial x^{2}}+\kappa u-U(x) v+\sigma|v|^{2} v=-i \gamma v,
\end{aligned}
$$

where $\gamma>0$ is the gain-loss coefficient. The $\mathcal{P} \mathcal{T}$ symmetry has been experimentally realized in photonics, and a large number of guided-wave-propagation regimes have been investigated in such systems [98-101]. In particular, as concerns solitons, although $\mathcal{P} \mathcal{T}$-symmetric systems belong to the class of dissipative ones, where solitons generally exist as isolated attractors, selected by the condition of the double balance between the dispersion (or diffraction) and nonlinearity, and between the gain and loss (the latter principle is very important for the creation of stable temporal solitons in fiber lasers [102]), in $\mathcal{P} \mathcal{T}$-symmetric systems solitons exist in continuous families, similar to their counterparts in conservative models $[100,101]$. In addition to the interest to fundamental studies, systems with the $\mathcal{P} \mathcal{T}$ symmetry offer promising applications, such as "light diodes", admitting unidirectional propagation of light in the waveguide, and lasers operating in the $\mathcal{P} \mathcal{T}$-symmetric regime [103].

\section{Annotation of Articles Included in the Special Issue}

The present Special Issue is composed of a collection of $\mathbf{2 0}$ contributions, which include $\mathbf{5}$ relatively brief reviews summarizing recently obtained results in various areas of the guided-wave propagation in photonics, and $\mathbf{1 5}$ original papers reporting novel findings in this broad field. The contributions may be naturally grouped according to different forms and manifestations of the guided-wave propagation addressed in these works. Accordingly, the list of papers published in the Special Issue (following below) is divided into 11 topics (A)-(K), and review articles are highlighted. In all cases, subjects addressed in the papers are sufficiently clearly defined by their titles.

(A) A batch of three papers may be classified as addressing problems arising in the fundamental (general) theory of the guided wave transmission in conservative (i.e., lossless) nonlinear media.

(A1) J. Fujioka, A. Gómez-Rodríguez, and Á. Espinosa-Cerón, Pulse Propagation Models with Bands of Forbidden Frequencies or Forbidden Wavenumbers: A Consequence of Abandoning the Slowly Varying Envelope Approximation and Taking into Account Higher-Order Dispersion. Appl. Sci. $2017,7,340$.

(A2) Chan, H.N.; Chow, K.W. Rogue Wave Modes for the Coupled Nonlinear Schrödinger System with Three Components: A Computational Study, Appl. Sci. 2017, 7, 559.

(A3) Govindarajan, A.; Malomed, B.A.; Mahalingam, A.; Uthayakumar, A. Modulational Instability in Linearly Coupled Asymmetric Dual-Core Fibers. Appl. Sci. 2017, 7, 645.

(B) A related topic is the study of bright and dark soliton in various settings. This topic is represented in the Special Issue by the following four contributions, one of them being a review article:

(B1) Mai, Z.; Xu, H.; Lin, F.; Liu, Y.; Fu, S.; Li, Y. Dark Solitons and Grey Solitons in Waveguide Arrays with Long-Range Linear Coupling Effects. Appl. Sci. 2017, 7, 311. 
(B2) Katsimiga, G.C.; Stockhofe, J.; Kevrekidis, P.G.; Schmelcher, P. Stability and Dynamics of Dark-Bright Soliton Bound States Away from the Integrable Limit. Appl. Sci. 2017, 7, 388.

(B3) Rodriguez, P.; Jimenez, J.; Guillet, T.; Ackemann, T. Polarization Properties of Laser Solitons. Appl. Sci. 2017, 7, 442.

(B4) Mitschke, R.F.; Mahnke, C.; Hause, A. Soliton Content of Fiber-Optic Light Pulses. Appl. Sci. 2017, 7, 635 .

(C) Specific aspects of transmission in optical waveguides are considered in the following three papers (the first two address problems of direct relevance to practical applications):

(C1) Lamy, M.; Finot, C.; Fatome, J.; Arocas, J.; Weeber, J.C.; Hammani, K. Demonstration of High-Speed Optical Transmission at $2 \mu \mathrm{m}$ in Titanium Dioxide Waveguides. Appl. Sci. 2017, 7, 63.

(C2) Memon, F.A.; Morichetti, F.; Melloni, A. Waveguiding Light into Silicon Oxycarbide. Appl. Sci. $2017,7,561$.

(C3) Morales, J.D.H.; Rodríguez-Lara, B.M. Photon Propagation through Linearly Active Dimers. Appl. Sci. 2017, 7, 587.

(D) Different aspects of the transmission of light in waveguides based on fiber Bragg gratings is considered in two papers:

(D1) Yang, S.-C.; He, Y.-J.; Wun, Y.-J. Designing a Novel High-Performance FBG-OADM Based on Finite Element and Eigenmode Expansion Methods. Appl. Sci. 2017, 7, 44.

(D2) Review: Liu, Y.; Fu, S.; Malomed, B.A.; Khoo, I.C.; Zhou, J. Ultrafast Optical Signal Processing with Bragg Structures. Appl. Sci. 2017, 7, 556.

(E) A specific phenomenon of bound states existing in the continuous spectrum of a waveguide built as an array of dielectric spheres is summarized in the following Review article:

Bulgakov, E.N.; Sadreev, A.F.; Maksimov, D.N. Light Trapping above the Light Cone in One-Dimensional Arrays of Dielectric Spheres. Appl. Sci. 2017, 7, 147.

(F) A topic of the propagation of self-accelerating beams in the form of Airy waves is overviewed in a Brief Review:

Zhang, Y.; Zhong, H.; Belić, M.R.; Zhang, Y. Guided Self-Accelerating Airy Beams-A Mini-Review. Appl. Sci. 2017, 7, 34.

(G) A specific aspect of the light propagation in metamaterials is considered in:

Mazzone, V.; Gongora, J.S.T.; Fratalocchi, A. Near-Field Coupling and Mode Competition in Multiple Anapole Systems. Appl. Sci. 2017, 7, 542.

(H) Some fundamental aspects of the light transmission in dissipative waveguides are addressed in the following paper:

Descalzi, O.; Cartes, C. Stochastic and Higher-Order Effects on Exploding Pulses. Appl. Sci. 2017, $7,887$.

(I) Theoretical studies of the propagation of light in $\mathcal{P} \mathcal{T}$-symmetric nonlinear waveguides are represented by an original paper,

D'Ambroise, J.; Kevrekidis, P.G. Existence, Stability and Dynamics of Nonlinear Modes in a 2D Partially P T Symmetric Potential. Appl. Sci. 2017, 7, 223.

(J) The propagation of plasmonic waves is addressed in the following two experimental works, with direct implications for applications:

(J1) Moon, K.; Lee, T.; Lee, Y.J.; Kwon, S. A Metal-Insulator-Metal Deep Subwavelength Cavity Based on Cutoff Frequency Modulation. Appl. Sci. 2017, 7, 86.

(J2) Iwanaga, M. Perfect Light Absorbers Made of Tungsten-Ceramic Membranes. Appl. Sci. 2017, $7,458$.

(K) Specific aspects of the general topic of fiber lasers, which are significant to fundamental and applied studies alike, are the subject of a Review article:

de Araújo, C.B.; Gomes, A.S.L.; Raposo, E.P. Lévy Statistics and the Glassy Behavior of Light in Random Fiber Lasers. Appl. Sci. 2017, 7, 644.

Conflicts of Interest: The author declares no conflicts of interest. 


\section{References}

1. Crisp, J.; Elliot, B. Introduction to Fiber Optics, 3rd ed.; Elsevier: Oxford, UK, 2005.

2. Agrawal, G.P. Fiber-Optic Communication Systems; John Wiley \& Sons: Hoboken, NJ, USA, 2010.

3. Poli, F.; Cucinotta, A. Photonic Crystal Fibers: Properties and Applications; Springer: Dordrecht, The Netherlands, 2007.

4. Yao, J. Microwave Photonics. J. Lightwave Technol. 2009, 27, 314-335.

5. Hutter, E.; Fendler, J.H. Exploitation of localized surface plasmon resonance. Adv. Mater. 2004, 16, $1685-1706$.

6. Maier, S.A.; Atwater, H.A. Plasmonics: Localization and guiding of electromagnetic energy in metal/dielectric structures. J. Appl. Phys. 2005, 98, 011101.

7. Gramotnev, D.K.; Bozhevolnyi, S.I. Plasmonics beyond the diffraction limit. Nat. Photonics 2010, 4, 83-91.

8. Savel'ev, S.; Yampol'skii, V.A.; Rakhmanov, A.L.; Nori, F. Terahertz Josephson plasma waves in layered superconductors: Spectrum, generation, nonlinear and quantum phenomena. Rep. Prog. Phys. 2010, 73, 026501.

9. Grimshaw, R.; Pelinovsky, E.; Talipova, T. Modelling internal solitary waves in the coastal ocean. Surveys Geophys. 2007, 28, 273-298.

10. Bongs, K.; Burger, S.; Dettmer, S.; Hellweg, D.; Arlt, J.; Ertmer, W.; Sengstock, K. Waveguide for Bose-Einstein condensates. Phys. Rev. A 2001, 63, 031602.

11. Lesanovsky, I.; von Klitzing, W. Time-averaged adiabatic potentials: Versatile matter-wave guides and atom traps. Phys. Rev. Lett. 2007, 99, 083001.

12. Khitun, A.; Bao, M.; Wang, K.L. Magnonic logic circuits. J. Phys. D Appl. Phys. 2010, 43, 264005.

13. Rigetti, C.; Gambetta, J.M.; Poletto, S.; Plourde, B.L.T.; Chow, J.M.; Corcoles, A.D.; Smolin, J.A.; Merkel, S.T.; Rozen, J.B.; Keefe, G.A.; et al. Superconducting qubit in a waveguide cavity with a coherence time approaching 0.1 ms. Phys. Rev. B 2012, 68, 100506.

14. Borisov, A.S.B.; Borovskiy, A.V.; Shriryaev, O.B.; Korovkin, W.; Prokhorov, A.M.; Solem, J.C.; Luk, S.T.; Boyer, K.; Rhodes, C.K. Relativistic and charge-displacement self-channeling of intense ultrashort laser-pulses in plasmas. Phys. Rev. A 1992, 45, 5830-5845.

15. Milchberg, H.M.; Durfee, C.G.; McIlrath, T.J. High-order frequency conversion in the plasma waveguide. Phys. Rev. Lett. 1995, 75, 2494-2497.

16. Spence, D.J.; Hooker, S.M. Investigation of a hydrogen plasma waveguide. Phys. Rev. E 2001, 63, 015401.

17. Snyder, A.W.; Love, J.D. Optical Waveguide Theory; Chapman \& Hall: London, UK, 1995.

18. Cronin, N.J. Microwave and Optical Waveguides; Institute of Physics Publishing: London, UK, 1995.

19. Zervas, M.N.; Codemard, C.A. High power fiber lasers: A review. IEEE J. Sel. Top. Quantum Electron. 2014, 20, 0904123.

20. Wright, L.G.; Christodoulides, D.N.; Wise, F. Controllable spatiotemporal nonlinear effects in multimode fibres. Nat. Photonics 2015, 9, 306.

21. Exner, P.; Kovař́k, H. Quantum Waveguides; Springer: Cham, Vietnam, 2015.

22. Olshanii, M. Atomic scattering in the presence of an external confinement and a gas of impenetrable bosons. Phys. Rev. Lett. 1998, 81, 938-941.

23. Paredes, B.; Widera, A.; Murg, V.; Mandel, O.; Folling, S.; Cirac, I.; Shlyapnikov, G.V.; Hansch, T.W.; Bloch, I. Tonks-Girardeau gas of ultracold atoms in an optical lattice. Nature 2004, 429, 277-281.

24. Bloch, I.; Dalibard, J.; Zwerger, W. Many-body physics with ultracold gases. Rev. Mod. Phys. 2008, 80, 885-964.

25. Snyder, A.W.; Mitchell, D.J.; Poladian, L.; Rowland, D.R.; Chen, Y. Physics of nonlinear fiber couplers. J. Opt. Soc. Am. B 1991, 8, 2101-2118.

26. Szameit, A.; Nolte, S. Discrete optics in femtosecond-laser-written photonic structures. J. Phys. B At. Mol. Opt. Phys. 2010, 43, 163001.

27. Garanovich, I.L.; Longhi, S.; Sukhorukov, A.A.; Kivshar, Y.S. Light propagation and localization in modulated photonic lattices and waveguides. Phys. Rep. 2012, 518, 1-79.

28. Fleischer, J.W.; Bartal, G.; Cohen, O.; Schwartz, T.; Manela, O.; Freedman, B.; Segev, M.; Buljan, H.; Efremidis, N.K. Spatial photonics in nonlinear waveguide arrays. Opt. Express 2005, 13, 1780-1796.

29. Lederer, F.; Stegeman, G.I.; Christodoulides, D.N.; Assanto, G.; Segev, M.; Silberberg, Y. Discrete solitons in optics. Phys. Rep. 2008, 463, 1-126.

30. Malomed, B.A. Variational methods in nonlinear fiber optics and related fields. Prog. Opt. 2002, 43, 71-193. 
31. Malomed, B.A. (Ed.) Spontaneous Symmetry Breaking, Self-Trapping, and Josephson Oscillations; Springer-Verlag: Berlin, Germany, 2013.

32. Zakharov, V.E.; Manakov, S.V.; Novikov, S.P.; Pitaevskii, L.P. Theory of Solitons; Nauka Publishers: Moscow, Russia, 1980. (In Russian); English Translation: New York, NY, USA, 1984.

33. Newell, A.C. Solitons and Inverse Scattering Transform; SIAM: Philadelphia, PA, USA, 1987.

34. Ablowitz, M.J.; Segur, H. Solitons and Inverse Scattering Transform; SIAM: Philadelphia, PA, USA, 2000.

35. Kevrekidis, P.G. The Discrete Nonlinear Schrödinger Equation: Mathematical Analysis, Numerical Computations, and Physical Perspectives; Springer: Berlin, Germany, 2009.

36. Hennig, D.; Tsironis, G.P. Wave transmission in nonlinear lattices. Phys. Rep. 1999, 307, 333-432.

37. Pitaevskii, L.P.; Stringari, S. Bose-Einstein Condensation; Clarendon: Oxford, UK, 2003.

38. Smith, H.; Pethick, C.J. Bose-Einstein Condensation in Dilute Gases, 2nd ed.; Cambridge University Press: Cambridge, UK, 2008.

39. Fibich, G. The Nonlinear Schrödinger Equation: Singular Solutions and Optical Collapse; Springer: Cham, Vietnam, 2015.

40. De Sterke, C.M.; Sipe, J.E. Gap solitons. Prog. Opt. 1994, 33, 203-260.

41. Aceves, A.B. Optical gap solitons: Past, present, and future; theory and experiments. Chaos 2000, 10, 584-589.

42. Eggleton, B.J.; Slusher, R.E.; de Sterke, C.M.; Krug, P.A.; Sipe, J.E. Bragg grating solitons. Phys. Rev. Lett. 1996, 76, 1627-1630.

43. Kashyap, R. Fiber Bragg Gratings, 2nd ed.; Academic Press: Burlington, MA, USA, 2009.

44. Etrich, C.; Lederer, F.; Malomed, B.A.; Peschel, T.; Peschel, U. Optical solitons in media with a quadratic nonlinearity. Prog. Opt. 2000, 41, 483-568.

45. Buryak, A.V.; di Trapani, P.; Skryabin, D.V.; Trillo, S. Optical solitons due to quadratic nonlinearities: From basic physics to futuristic applications. Phys. Rep. 2002, 370, 63-235.

46. Chin, C.; Grimm, R.; Julienne, P.; Tiesinga, E. Feshbach resonances in ultracold gases. Rev. Mod. Phys. 2010, $82,1225-1285$.

47. Kivshar, Y.S.; Agrawal, G.P. Optical Solitons: From Fibers to Photonic Crystals; Academic Press: San Diego, CA, USA, 2003.

48. Kharif, C.; Pelinovsky, E. Physical mechanisms of the rogue wave phenomenon. Eur. J. Mech. B Fluids 2003, 22, 603-634.

49. Onorato, M.; Residori, S.; Bortolozzo, U.; Montina, A.; Arecchi, F.T. Rogue waves and their generating mechanisms in different physical contexts. Phys. Rep. 2013, 528, 47-89.

50. Vachaspati, T. Kinks and Domain Walls; Cambridge University Press: Cambridge, UK, 2006.

51. Rajaraman, R. Solitons and Instantons; North Holland: Amsterdam, The Netherlands, 1982.

52. Dauxois, T.; Peyrard, M. Physics of Solitons; Cambridge University Press: Cambridge, UK, 2006.

53. Strecker, K.E.; Partridge, G.B.; Truscott, A.G.; Hulet, A.G. Bright matter wave solitons in Bose-Einstein condensates. New J. Phys. 2003, 5, 73.1-73.8.

54. Cerda-Méndez, E.A.; Sarkar, D.; Krizhanovskii, D.N.; Gavrilov, S.S.; Biermann, K.; Skolnick, M.S.; Santos, P.V. Exciton-polariton gap solitons in two-dimensional lattices. Phys. Rev. Lett. 2013, 111, 146401.

55. Petviashvili, V.I.; Pokhotelov, O.A. Solitary Waves in Plasmas and Atmosphere; Routledge: London, UK, 1997.

56. Bar'yakhtar, V.G.; Chetkin, M.V.; Ivanov, B.A.; Gadetskii, S.N. Dynamics of Topological Magnetic Solitons; Springer-Verlag: Berlin, Germany, 1994.

57. Ustinov, A.V. Solitons in Josephson junctions. Phys. D 1998, 123, 315-329.

58. Naugolnykh, K.; Ostrovsky, L. Nonlinear Wave Processes in Acoustics; Cambridge University Press: Cambridge, UK, 1998.

59. Silberberg, Y. Collapse of optical pulses. Opt. Lett. 1990, 15, 1282-1284.

60. Malomed, B.A.; Mihalache, D.; Wise, F.; Torner, L. Spatiotemporal Optical Solitons. J. Opt. B Quantum Semicl. Opt. 2005, 7, R53-R72.

61. Malomed, B.A.; Mihalache, D.; Wise, F.; Torner, L. Viewpoint: On multidimensional solitons and their legacy in contemporary Atomic, Molecular and Optical physics. J. Phys. B At. Mol. Opt. Phys. 2016, 49, 170502.

62. Malomed, B.A. Multidimensional solitons: Well-established results and novel findings. Eur. Phys. J. Spec. Top. 2016, 25, 2507-2532. 
63. Minardi, S.; Eilenberger, F.; Kartashov, Y.V.; Szameit, A.; Röpke, J.; Kobelke, J.; Schuster, K.; Bartelt, H.; Nolte, S.; Torner, L.; et al. Three-dimensional light bullets in arrays of waveguides. Phys. Rev. Lett. 2010, 105, 263901.

64. Eilenberger, F.; Prater, K.; Minardi, S.; Geiss, R.; Röpke, U.; Kobelke, J.; Schuster, K.; Bartelt, H.; Nolte, S.; Tünnermann, A.; et al. Observation of discrete, vortex light bullets. Phys. Rev. X 2013, 3, 041031.

65. Cuevas, J.; Kevrekidis, P.G.; Malomed, B.A.; Dyke, P.; Hulet, R. Interactions of solitons with a Gaussian barrier: Splitting and recombination in quasi-1D and 3D. New J. Phys. 2013, 15, 063006.

66. Joannopoulos, J.D.; Johnson, S.G.; Winn, J.N.; Meade, R.D. Photonic Crystals: Molding the Flow of Light, 2nd ed.; Princeton University Press: Princeton, NJ, USA, 2008.

67. Poddubny, A.N.; Ivchenko, E.L. Photonic quasicrystalline and aperiodic structures. Physica E 2010, 42, 1871-1895.

68. Vardeny, Z.V.; Nahata, A.; Agrawal, A. Optics of photonic quasicrystals. Nat. Photonics 2013, 7, 177-187.

69. Istrate, E.; Sargent, E.H. Photonic crystal heterostructures and interfaces. Rev. Mod. Phys. 2006, 78, 455-481.

70. Benabid, F.; Roberts, P.J. Linear and nonlinear optical properties of hollow core photonic crystal fiber. J. Mod. Opt. 2011, 58, 87-124.

71. Russell, P.S.J.; Hölzer, P.; Chang, W.; Travers, A.A.A.C. Hollow-core photonic crystal fibres for gas-based nonlinear optics. Nat. Photonics 2014, 8, 278-286.

72. Saleh, M.F.; Biancalana, F. Soliton dynamics in gas-filled hollow-core photonic crystal fibers. J. Opt. 2016, 18,013002 .

73. Johnson, S.G.; Ibanescu, M.; Skorobogatiy, M.; Weisberg, O.; Engeness, T.; Soljačić, M.; Jacobs, S.A.; Joannopoulos, J.D.; Fink, Y. Low-loss asymptotically single-mode propagation in large-core OmniGuide fibers. Opt. Exp. 2001, 9, 748-779.

74. Kartashov, Y.V.; Malomed, B.A.; Torner, L. Solitons in nonlinear lattices. Rev. Mod. Phys. 2011, 83, $247-306$.

75. Sakaguchi, H.; Malomed, B.A. Solitons in combined linear and nonlinear lattice potentials. Phys. Rev. A 2010, $81,013624$.

76. Borovkova, O.V.; Kartashov, Y.V.; Torner, L.; Malomed, B.A. Bright solitons from defocusing nonlinearities. Phys. Rev. E 2011, 84, 035602.

77. Kartashov, Y.V.; Malomed, B.A.; Shnir, Y.; Torner, L. Twisted toroidal vortex-solitons in inhomogeneous media with repulsive nonlinearity. Phys. Rev. Lett. 2014, 113, 264101.

78. Kinoshita, S.; Yoshioka, S.; Miyazaki, J. Physics of structural colors. Rep. Progr. Phys. 2008, 17, 076401.

79. Shalaev, V.M. Optical negative-index metamaterials. Nat. Photonics 2007, 1, 41-48.

80. Soukoulis, C.M.; Wegener, M. Past achievements and future challenges in the development of three-dimensional photonic metamaterials. Nat. Photonics 2011, 5, 523-530.

81. Jacob, Z.; Alekseyev, L.V.; Narimanov, E. Optical hyperlens: Far-field imaging beyond the diffraction limit. Opt. Exp. 2006, 14, 8247-8256.

82. Cai, W.; Chettiar, U.K.; Kildishev, A.V.; Shalaev, V.M. Optical cloaking with metamaterials. Nat. Photonics 2007, 1, 224-227.

83. Drachev, V.P.; Podolskiy, V.A.; Kildishev, A.V. Hyperbolic metamaterials: New physics behind a classical problem. Opt. Exp. 2013, 21, 15048-15064.

84. Poddubny, A.; Iorsh, I.; Belov, P.; Kivshar, Y. Hyperbolic metamaterials. Nat. Photonics 2013, 7, $958-967$.

85. Kildishev, A.V.; Boltasseva, A.; Shalaev, V.M. Planar Photonics with Metasurfaces. Science 2013, 339, 1232009.

86. Chen, H.-T.; Taylor, A.J.; Yu, N. A review of metasurfaces: physics and applications. Rep. Prog. Phys. 2016, $79,076401$.

87. Liberal, I.; Engheta, N. Near-zero refractive index photonics. Nat. Photonics 2017, 11, 149-159.

88. Rechtsman, M.C.; Zeuner, J.M.; Plotnik, Y.; Lumer, Y.; Podolsky, D.; Dreisow, F.; Nolte, S.; Segev, M.; Szameit, A. Photonic Floquet topological insulators. Nature 2013, 496, 196-200.

89. Kartashov, Y.V.; Skryabin, D.V. Modulational instability and solitary waves in polariton topological insulators. Optica 2016, 3, 1228-1236.

90. Lu, L.; Joannopoulos, J.D.; Soljačić, M. Topological photonics. Nat. Photonics 2014, 8, 821-829.

91. Maimistov, A.I.; Gabitov, I.R. Nonlinear optical effects in artificial materials. Eur. Phys. J. Spec. Top. 2007, 147, 265-286.

92. Baeva, A.; Prasad, P.N.; Grenb, H.Ȧ.; Samoć, M.; Wegener, A. Metaphotonics: An emerging field with opportunities and challenges. Phys. Rep. 2015, 594, 1-60. 
93. Urbas, A.M.; Jacob, Z.; Dal Negro, L.; Engheta, N.; Boaedman, A.D.; Egan, P.; Khanikaec, A.B.; Menon, V.; Ferrera, M.; Kinsey, N.; et al. Roadmap on optical metamaterials. J. Opt. 2016, 18, 093005.

94. Garnett, E.; Yang, P. Light trapping in silicon nanowire solar cells. Nano Lett. 2010, 10, 1082-1087.

95. Kartashov, Y.V.; Konotop, V.V.; Vysloukh, V.A.; Zezyulin, D.A. Guided modes and symmetry breaking supported by localized gain. In Spontaneous Symmetry Breaking, Sepf-Trapping, and Josephson Oscillations; Malomed, B.A., Ed.; Springer-Verlag: Berlin, Germany, 2013; pp. 149-166.

96. Malomed, B.A. Spatial solitons supported by localized gain. J. Opt. Soc. Am. B 2014, 31, 2460-2475.

97. Lobanov, V.E.; Borovkova, O.V.; Malomed, B.A. Dissipative quadratic solitons supported by a localized gain. Phys. Rev. A 2014, 90, 053820.

98. Makris, K.G.; El-Ganainy, R.; Christodoulides, D.N.; Musslimani, Z.H. $\mathcal{P} \mathcal{T}$-Symmetric Periodic optical potentials. Int. J. Theor. Phys. 2011, 50, 1019-1041.

99. Bender, C.M.; DeKieviet, M.; Klevansky, S.P. PT quantum mechanics. Phil. Trans. R. Soc. A Math. Phys. Eng. Sci. 2013, 371, 20120523.

100. Sukhorukov, S.V.S.A.A.; Huang, J.; Dmitriev, S.V.; Lee, C.; Kivshar, Y.S. Nonlinear switching and solitons in $\mathcal{P} \mathcal{T}$-symmetric photonic systems. Laser Photonics Rev. 2016, 10, 177.

101. Konotop, V.V.; Yang, J.; Zezyulin, D.A. Nonlinear waves in $\mathcal{P} \mathcal{T}$-symmetric systems. Rev. Mod. Phys. 2016, 88, 035002.

102. Grelu, P.; Akhmediev, N. Dissipative solitons for mode-locked lasers. Nat. Photonics 2012, 6, 84-92.

103. Huang, Y.; Shen, Y.; Min, C.; Fan, S.H.; Veronić, G. Unidirectional reflectionless light propagation at exceptional points. Nanophotonics 2017, 6, 977-996.

(C) 2017 by the authors. Licensee MDPI, Basel, Switzerland. This article is an open access article distributed under the terms and conditions of the Creative Commons Attribution (CC BY) license (http:/ / creativecommons.org/licenses/by/4.0/). 